\title{
BMJ Open Barriers and enablers in primary care clinicians' management of osteoarthritis: protocol for a systematic review and qualitative evidence synthesis
}

\author{
T Egerton, ${ }^{1}$ L Diamond, ${ }^{1}$ R Buchbinder,${ }^{2,3}$ K Bennell, ${ }^{1}$ S C Slade ${ }^{2,3}$
}

To cite: Egerton T, Diamond L, Buchbinder R, et al. Barriers and enablers in primary care clinicians' management of osteoarthritis: protocol for a systematic review and qualitative evidence synthesis. BMJ Open 2016;6: e011618. doi:10.1136/ bmjopen-2016-011618

- Prepublication history and additional material is available. To view please visit the journal (http://dx.doi.org/ 10.1136/bmjopen-2016011618).

Received 22 February 2016 Revised 20 April 2016 Accepted 9 May 2016

\section{(1) crossank}

${ }^{1}$ Centre for Health Exercise \& Sports Medicine, Medicine, Dentistry \& Health Sciences, The University of Melbourne, Melbourne, Victoria, Australia ${ }^{2}$ Department of Epidemiology and Preventive Medicine, School of Public Health \& Preventive Medicine, Monash University, Melbourne, Victoria, Australia

${ }^{3}$ Monash Department of Clinical Epidemiology, Cabrini Hospital, Melbourne, Victoria, Australia

Correspondence to

Dr T Egerton;

thor@sutmap.com

\section{ABSTRACT}

Introduction: Osteoarthritis is a highly prevalent and disabling condition. Primary care management of osteoarthritis is generally suboptimal despite evidence for several modestly effective interventions and the availability of high-quality clinical practice guidelines. This report describes a planned study to synthesise the views of primary care clinicians on the barriers and enablers to following recommended management of osteoarthritis, with the aim of providing new interpretations that may facilitate the uptake of recommended treatments, and in turn improve patient care.

Methods and analysis: A systematic review and meta-synthesis of qualitative studies. 5 databases will be searched using key search terms for qualitative research, evidence-based practice, clinical practice guidelines, osteoarthritis, beliefs, perceptions, barriers, enablers and adherence. A priori inclusion/exclusion criteria include availability of data from primary care clinicians, reports on views regarding management of osteoarthritis, and studies using qualitative methods for both data collection and analysis. At least 2 independent reviewers will identify eligible reports, conduct a critical appraisal of study conduct, extract data and synthesise reported findings and interpretations. Synthesis will follow thematic analysis within a grounded theory framework of inductive coding and iterative theme identification.

The reviewers plus co-authors will contribute to the meta-synthesis to find new themes and theories. The Confidence in the Evidence from Reviews of Qualitative research (CERQual) approach will be used to determine a confidence profile of each finding from the meta-synthesis. The protocol has been registered on PROSPERO and is reported using the Preferred Reporting Items for Systematic Review and MetaAnalyses Protocols (PRISMA-P) guidelines.

Ethics and dissemination: Ethical approval is not required. The systematic review will be published in a peer-reviewed journal. The results will help to inform policy and practice and assist in the optimisation of management for people with osteoarthritis.

PROSPERO registration number: CRD42015027543.
Strengths and limitations of this study

- The search strategy is designed to be comprehensive and informed by Cochrane review methods, published recommendations for optimal qualitative research identification, and a previously published protocol.

- Inclusion/exclusion criteria and data extraction have been determined a priori to reduce bias in sourcing data, and study screening, data extraction, critical appraisal of study conduct and data analysis will be independently conducted by more than one reviewer with a further reviewer available for arbitration to reach consensus.

- The first limitation is removal of data from the original contexts and the participant quotes are no longer linked to the context of the original questions or the participant's setting.

- The second limitation is that the synthesis relies on the data presented in each of the included reports which may not reflect the full analysis of the original data.

- The inclusion of only English language publications means there is potential for cultural and publication bias in the findings.

\section{INTRODUCTION}

Osteoarthritis (OA) is a major global public health problem ${ }^{1}$ causing significant pain and disability, and is now ranked 13th in global causes of years lived with disability. ${ }^{2}$ There is no cure, but an extensive body of research has provided evidence to support use of a range of modestly effective treatments for symptom and function management. ${ }^{3-6}$ Evidence-based and expert consensus-based clinical practice guidelines (CPGs) have been produced to provide recommendations for effective treatments and best practice for OA management. ${ }^{7-13}$ Across these CPGs, conservative non-drug non-surgical care is advocated as the cornerstone for management of OA in all joints. This care includes 
education about the disease process, pain mechanisms and treatment options, and promotion of selfmanagement with emphasis on positive behavioural changes, in particular exercise for all patients and weight loss for overweight or obese patients, ${ }^{10}$ regardless of joint(s) affected. Pharmacological options most recommended include acetaminophen/paracetamol as first-line, and non-steroidal anti-inflammatory drugs (topical or oral) as second-line options. Guidelines for management of knee OA uniformly advise against the use of arthroscopic debridement and/or lavage. ${ }^{8} 913$ Total knee or hip arthroplasty is recommended when people have severe symptomatic knee or hip OA. ${ }^{10} 14$

While there is a large amount of agreement and overlap across recommendations in CPGs, some inconsistencies are apparent. ${ }^{14}$ Inconsistencies may occur because of the specific focus of the guideline, because of variations in quality and rigour of guideline development procedures, and because of changes in evidence over time. For example, recommendations have been specifically produced for management of hand $\mathrm{OA},{ }^{11}$ while others include all OA. ${ }^{9}$ Guidelines have also been produced which focus only on non-surgical ${ }^{8} 15$ or nonpharmacological ${ }^{12}$ management of OA. Quality assessment using the Appraisal of Guidelines Research and Evaluation (AGREE) Instrument $^{16}$ indicates suboptimal quality of many CPGs for OA. ${ }^{14}$

Guidelines use a range of evidence to formulate recommendations with high-quality evidence used where it exists but where it is lacking, recommendations may be based on lower quality evidence and/or expert opinion. Despite a lack of high-quality evidence, walking aids and thermal modalities are widely endorsed by CPGs for OA, while acupuncture, knee braces, heel wedges, intra-articular hyaluronans, glucosamine and chondroitin remain controversial. With time, evidence for or against some existing recommendations strengthens and evidence to support new options emerges. For example, the recommendation around the use of imaging for OA diagnosis has evolved, with older guidelines often recommending plain film X-ray to assist in the diagnosis of OA, while the recent National Institute for Health and Care Excellence (NICE) guidelines recommend that $\mathrm{OA}$ should be diagnosed clinically and without imaging. ${ }^{9}$

$\mathrm{OA}$ is mostly diagnosed and managed in primary care settings ${ }^{17} 18$ and mostly by general practitioners (GPs; ie, family doctors). Some conservative interventions with recommendations for their use in CPGs, in particular exercise, weight loss, pain management advice and provision of other joint support or protection devices, may also be provided in primary care by allied health practitioners including physiotherapists, occupational therapists, exercise physiologists, podiatrists, pharmacists, practice nurses, dieticians and nutritionists. Survey and questionnaire data have shown that care received by individuals with $\mathrm{OA}$ in primary care settings is often inconsistent with broad CPG recommendations. ${ }^{15}$ 19-24
Suboptimal care has been demonstrated across a number of quality domains including provision of effective treatments, safety, access to educational material and support for self-management. ${ }^{22}$ For example, the Australian CareTrack study showed that only $43 \%$ of people with OA received recommended care. ${ }^{20}$ In particular, non-drug, non-surgical interventions are not given the importance by GPs that is recommended by all CPGs, ${ }^{15} 2324$ while prescribing patterns appear to be better aligned with recommendations for pharmacological treatments. ${ }^{25}{ }^{26}$ However, one study found there may be higher levels of prescription of more potent opioids than evidence suggests is warranted. ${ }^{15}$

The development and dissemination of CPGs has been suggested as one method for improving the alignment of practice with evidence for effectiveness. However, previous research has highlighted that the availability of CPGs does not necessarily lead to evidence-based practice. ${ }^{1527}$ Variations in accessing and uptake of research evidence and CPGs occur between different clinicians, between different sources of evidence and between different recommendations within guidelines. $^{27}$ Clinician variations occur for many reasons, from differences in preferences for accessing information to strength of personal beliefs about health interventions. How concrete a recommendation is written, how complex or difficult the procedure is to provide, how credible the recommendation seems, and how feasible it is to implement locally will also influence uptake. $^{27} 28$ These challenges are experienced across many health disorders where CPGs have been produced. With or without CPGs, change to more effective practice is often slow and inconsistent. ${ }^{28-31}$

Qualitative methodologies are appropriate for exploring the nature of perceptions, beliefs, barriers and enablers that can influence whether practices align to evidence and recommendations. ${ }^{32}{ }^{33}$ Qualitative synthesis of primary qualitative studies can pull together findings from across different settings and generate new theoretical or conceptual models. ${ }^{34}$ We have planned a qualitative synthesis ${ }^{35-37}$ exploring the barriers, enablers and/or beliefs and perceptions that may act as barriers or enablers to implementation of effective treatments and/or CPG recommendations for OA within the context of primary care practice. The findings may be helpful in informing our understanding of the complexity of implementing evidence-based guidelines for OA management and lead to innovations in addressing the evidence practice gap.

\section{METHODS}

The study has been registered on PROSPERO (http:// www.crd.york.ac.uk/PROSPERO; 4/11/2015, registration number CRD42015027543). The reporting of this protocol is in accordance with the Preferred Reporting Items for Systematic Reviews and Meta-Analyses (PRISMA) Statement for Protocols (PRISMA-P checklist) ${ }^{38}$ (see 
online supplementary appendix 1), and the Enhancing Transparency in Reporting the synthesis of Qualitative research (ENTREQ) checklist where relevant to a protocol (see online supplementary appendix 1). ${ }^{34}$ The methods are informed by a meta-synthesis of barriers and enablers in clinician adherence to guidelines for low back pain ${ }^{39}$ and Cochrane review methods. ${ }^{40}{ }^{41}$ The reporting of the final review will also be according to PRISMA and ENTREQ recommendations.

\section{Eligibility criteria}

Articles that report empirical data from primary care clinicians who provide treatments for OA (GPs or family doctors, physiotherapists, occupational therapists, exercise physiologists, podiatrists, pharmacists, practice nurses, dieticians and nutritionists) will be included. Views on barriers, enablers, beliefs and/or perceptions regarding management of $\mathrm{OA}$ that were based on $\mathrm{OA}$ CPG recommendations will be sought. Studies will be excluded if they did not utilise qualitative methods for data collection and analysis. Mixed-methods studies will be included if the qualitative data are reported separately. Studies with multiple participant cohorts will be included if eligible primary care clinician groups' data are reported separately. Articles reporting on other types of arthritis will be included only if data on OA are reported separately. Only English language reports will be included.

\section{Identification and selection of studies}

The search strategy was based on the one published for MEDLINE by Slade $e t a l^{39}$ and adapted for OA and other databases. The strategy aims to identify all relevant articles published in peer-reviewed journals, and thus be a comprehensive assembly of the current state of knowledge around the topic. Five electronic databases including MEDLINE, The Cochrane Central Register of Controlled Trials, EMBASE, CINAHL and PsychInfo will be searched from inception to October 2015 (see online supplementary appendix 2 for the MEDLINE search strategy). The five databases were selected to optimise likelihood of identifying all the previously published studies while maintaining a manageable screening load. The search uses explosions and combinations of key search terms for qualitative research, evidence-based practice, CPGs, OA, beliefs, perceptions, barriers, enablers and adherence. Search results will be collated in a reference database (Endnote V.X7), duplicates deleted and then initial screening of titles will be independently conducted by two reviewers (TE and LD). A priori inclusion/exclusion criteria will be applied at this stage (see online supplementary appendix 3). The two reviewers will then independently screen abstracts of any titles retained by at least one reviewer. Inclusion/ exclusion criteria will be independently applied by two reviewers to full texts of remaining references to select the final studies to include in the review. Manual searching of the reference lists and citation tracking of papers identified as potentially relevant at this stage will also be conducted. Discrepancies in the final decision on inclusion will be discussed and if necessary reviewed by a third reviewer (SCS) in order to reach consensus. Study selection will be documented and summarised in a PRISMA compliant flow chart. The process of identification and selection of articles and flow chart of article inclusion is outlined in figure 1 .

\section{Data extraction}

Data extracted from the articles will include study details (location and setting, population, research question/ aims, guidelines the interview questions were developed from, data collection method, data analysis method, theoretical framework) and findings (barriers, enablers, other beliefs or perspectives, second-order themes or interpretations and subthemes, supporting quotations, conclusions and recommendations; see online supplementary appendix 4A). Quotations from primary study participants (first-order data) and quotes from report authors (second-order data) will be assembled retaining links to contextual information and the findings of the assessment of quality of conduct and reporting from the original study. The extracted themes from each of the primary studies will be considered for common domains and dissonant cases. In studies where participants included patients or health professionals other than eligible primary care clinicians (eg, surgeons or rheumatologists), only data that could be attributed to the eligible primary care clinicians will be extracted. In studies that used mixed methods such as quantitative surveys, only data from qualitative components of the report will be extracted. Two reviewers (TE and LD) will independently extract the data into spreadsheets (Microsoft Excel) and discrepancies will be resolved, by consensus, when the two data sets are merged.

\section{Critical appraisal of conduct of included studies}

The Critical Appraisal Skills Programme (CASP) checklist $^{42}$ will be used to identify and appraise methodological quality (trustworthiness) of the included studies independently by two raters (TE and LD). Assessment of trustworthiness in qualitative research considers study rigour, or the thoroughness and appropriateness of conduct; credibility, or whether the findings were data driven and meaningful; and relevance of the research question to the review topic. ${ }^{43}$ The CASP checklist of 10 questions comes with some decision rules and guidance on the interpretation of each item. Using the example of Slade et $a l,{ }^{44}$ the review team will construct an expanded summary table detailing the presence or absence of the components of each CASP question (see online supplementary appendix $4 \mathrm{~B}$ ). A summary narrative report of the trustworthiness of the individual included studies will be provided. Summary scores or quality ratings will not be generated as the CASP does not have a scoring matrix and a cut-off point has not been established for ratings of quality of qualitative 
Figure 1 Flow diagram of study identification and selection, adapted from PRISMA flow chart. PRISMA, Preferred Reporting Items for Systematic Review and Meta-Analysis.

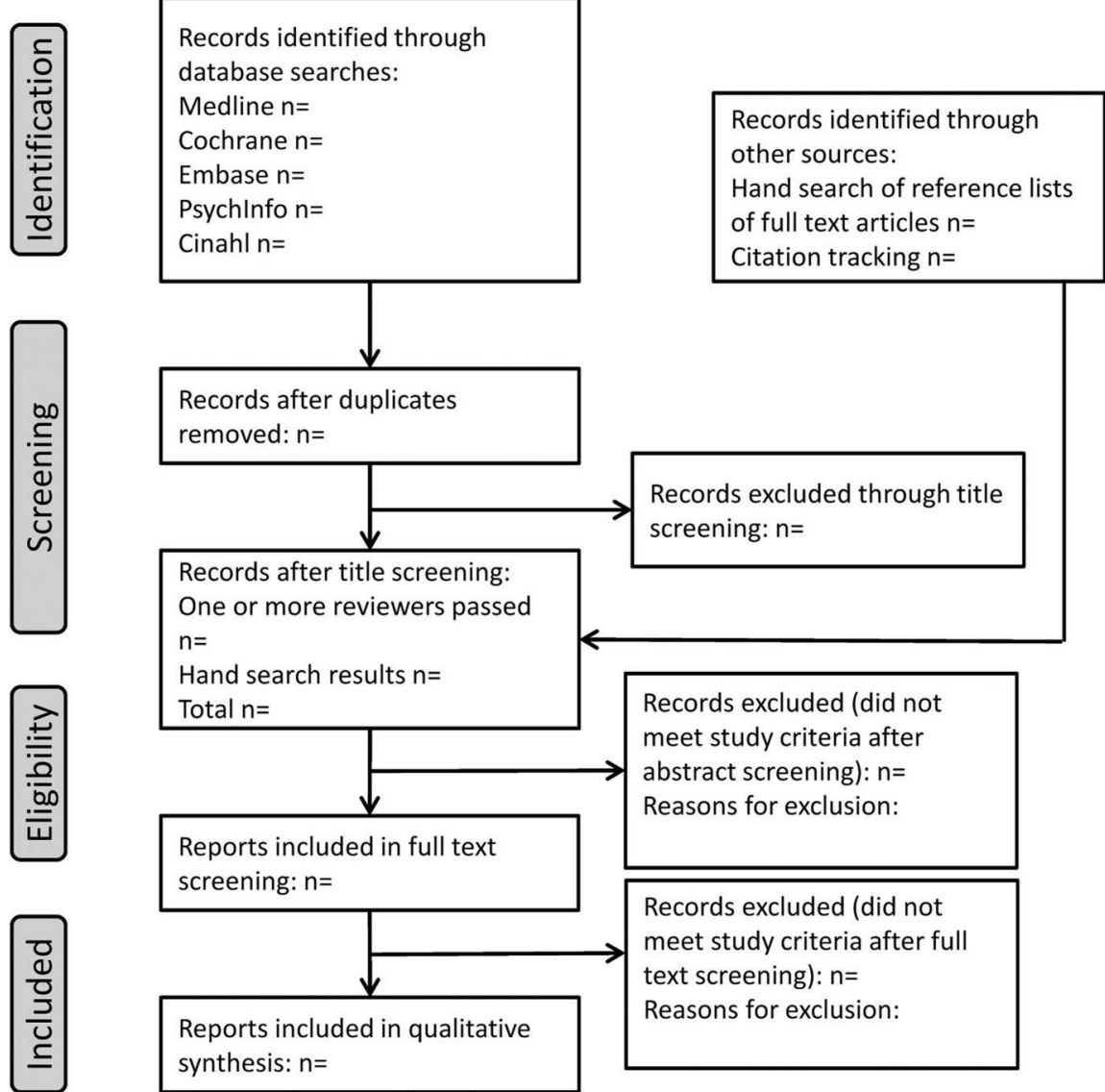

studies. Studies will not be excluded from the review on the basis of the critical appraisal of conduct and we will discuss the impact on the data synthesis of any study weaknesses.

\section{Data synthesis and higher order theme and theory development}

A meta-synthesis approach will be used for this qualitative data synthesis, ${ }^{35-37}$ and underpinned by the philosophical positioning that knowledge of reality is mediated by one's beliefs and perceptions. It is a systematic and comprehensive approach whereby data are coded and organised into descriptive themes, from which new higher order themes are developed that offer new interpretations beyond the primary studies' findings. ${ }^{35-37}$

The first stage of data synthesis will be to assemble and simplify the primary data, themes and subthemes into common groups within an external framework of barriers, enablers and other beliefs/perceptions that can act as barriers or enablers. After familiarisation with the data, recurrent codes for meaning and content across studies will be identified. Development of the codes (and a coding framework if possible) and the actual coding of the data set will be conducted by three coders (SCS, TE and LD) through discussions and iterations. All reviewers will consider all the available data. During discussions and coding rounds, ideas will be shared and refined until a final set of themes is agreed by consensus. The text to which a given code was applied will be checked for consistency of interpretation and to further refine the themes. Bearing in mind each of the broad concepts of barriers, enablers and other beliefs/perceptions relating to $\mathrm{OA}$ management, higher order themes will be inductively derived from the patterns in the codes. These themes may or may not have been identified by the primary study authors. Where possible, new major higher order themes will retain key contextual factors and new overarching or expanded theory will be developed through discussion between the reviewers and co-researchers. This stage relies on the judgement, insight and creativity of thinking among the researchers but will be anchored to the empirical data and review question using a grounded theory framework. ${ }^{32} 33$ Interpretation of findings will consider new understandings in relation to factors known to influence guidelines adherence: clinician knowledge, professional background, cultural factors, environmental factors and patient drivers; and the main CPG recommendations for knee OA management related to education, selfmanagement support for exercise and weight loss, firstline and second-line pharmacological management, and appropriate use of surgical interventions.

\section{Assessment of credibility and rigour of new findings}

The robustness of the synthesis and confidence in the review findings, defined as the analytical output of the evidence synthesis of the primary studies, will be determined as a final stage using the Confidence in the 
Evidence from Reviews of Qualitative research (CERQual) approach. ${ }^{45}$ This approach includes consideration of (1) methodological limitations (design and conduct flaws) of each primary research study contributing to the finding and how the limitations might affect confidence in the finding; (2) relevance of the included studies to the overall review question in terms of, for example, the population, setting and original study aim; (3) coherence and consistency of results across the included studies and (4) adequacy of supporting data (quantity as well as quality). In this way, quality of the empirical studies will influence the emphasis given to data informing themes and any new theories that are generated. The CERQual is not a critical appraisal of the methodological limitations of either individual studies or the evidence synthesis, nor does it assess confidence in the overall synthesis findings, but considers each new finding separately. Confidence judgements will be achieved through discussion between at least two of the review authors. Each review finding will be allocated a level of confidence. Confidence levels start at 'high confidence' and are rated down by one or more levels if there are concerns regarding any of the individual CERQual components. Results will be summarised along with the main findings textually and in a table adapted from the CERQual Qualitative Evidence Profile table ${ }^{45}$ with the following headings:

- Individual review finding;

- Studies contributing to the review finding;
- Methodological limitations-problems with the design or conduct or reporting of primary studies;

- Relevance-applicable to context and degree to which the finding is generalisable;

- Coherence-grounding in the primary data with evidence provided by quotations from primary study participants;

- Adequacy of data-detail, depth and amount of supporting data;

- Overall CERQual assessment of confidence-four levels of confidence in the evidence for an individual review finding: high, moderate, low or very low;

- Explanation of confidence judgement.

The proposed step-by-step procedure for the data extraction and meta-synthesis is shown in figure 2.

\section{DISCUSSION}

This planned study is a systematic review and metasynthesis that will use rigorous and explicit methods ${ }^{40} 41$ to bring together the results of empirical qualitative studies investigating perceptions and beliefs, barriers and enablers to practice based on CPG recommendations for the management of OA by primary care clinicians. The purpose is to synthesise the primary data to provide new interpretations that may assist in identification of strategies with the potential for facilitating uptake of effective treatments and CPG recommendations.
Figure 2 Model of the proposed meta-synthesis derived from Cochrane review methods, metasynthesis methodology and CERQual approach. CASP, Critical Appraisal Skills Programme; CERQual, Confidence in the Evidence from Reviews of Qualitative research.

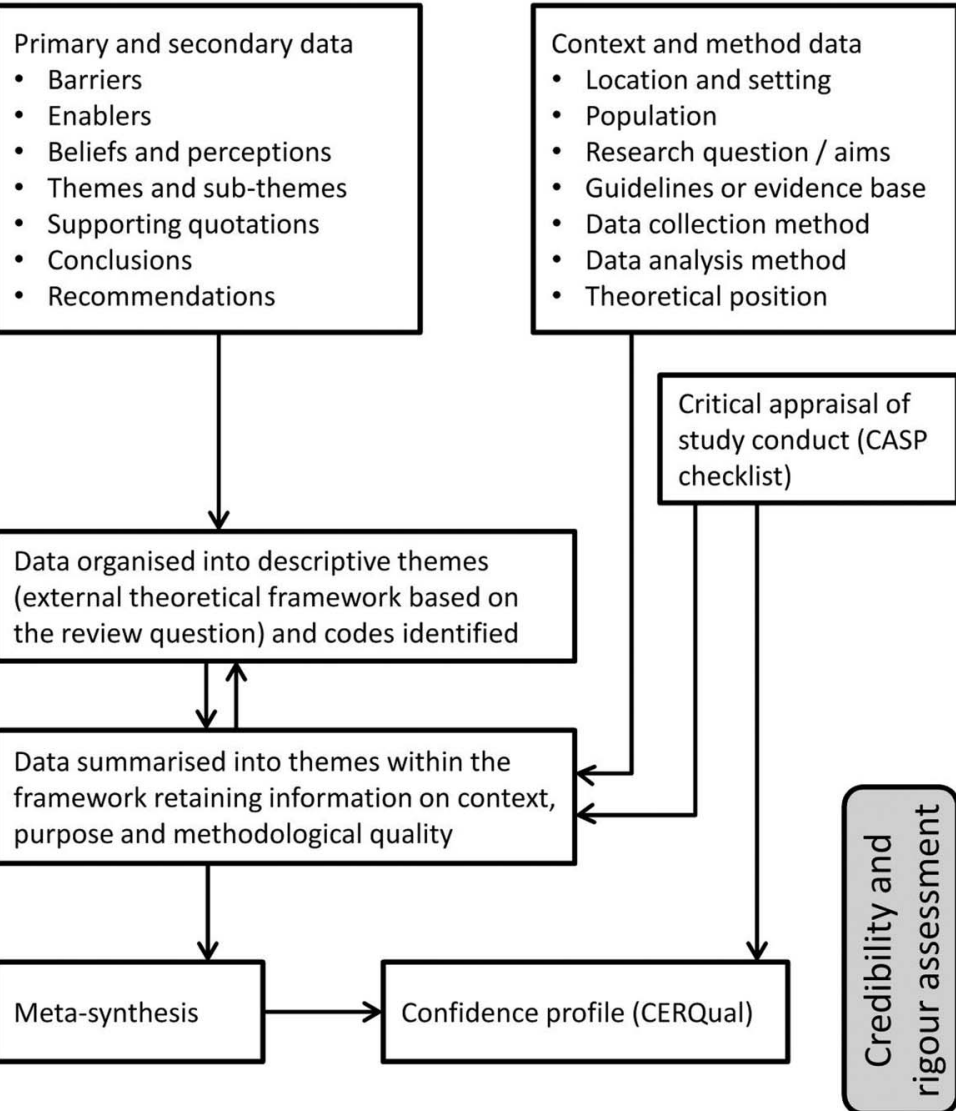


In-depth understanding of the barriers and enablers to achieving effective practice is needed in order to bridge the gap between research findings and clinical practice. Interventions which might include behaviour change interventions, service delivery changes and/or others can then be specifically designed to address the barriers unique to the population and the target practice. ${ }^{46-49}$ Implementation interventions tailored to identify barriers may be more likely to improve practice than more general interventions. ${ }^{50}$ Implementing consistent and evidence-based management for patients with OA may alleviate some confusion and frustration for patients and providers, lead to better health outcomes and possibly reduce healthcare costs.

This protocol paper serves to predefine our objectives and methods and also to communicate our intent. Any deviations between this protocol and our actual methods will be discussed in the systematic review report. It is anticipated that through the assimilation and interpretation of the attitudes and experiences of primary care clinicians managing OA, reported by studies that may vary in original purpose and context, we will develop a richer understanding of the potential barriers, enablers and beliefs or perceptions that may act as barriers or enablers to optimal management of $\mathrm{OA}$ and assist in future policy and service delivery improvements.

Contributors TE and KB conceived the idea for the study. TE, SCS and RB were responsible for the study design and protocol. TE drafted the protocol manuscript with input from LD, SCS, RB and KB. All authors have read and approved the final manuscript. The corresponding author guarantees the paper and that the authorship statement is correct.

Funding This research received funding from National Health \& Medical Research Council (NHMRC) Centre of Research Excellence (CRE) \#1079078. TE is funded by the CRE. RB is funded by an NHMRC Senior Principal Research Fellowship and KB is funded by an NHMRC Principal Research Fellowship.

Competing interests None declared

Provenance and peer review Not commissioned; externally peer reviewed.

Open Access This is an Open Access article distributed in accordance with the Creative Commons Attribution Non Commercial (CC BY-NC 4.0) license, which permits others to distribute, remix, adapt, build upon this work noncommercially, and license their derivative works on different terms, provided the original work is properly cited and the use is non-commercial. See: http:// creativecommons.org/licenses/by-nc/4.0/

\section{REFERENCES}

1. Murray CJ, Barber RM, Foreman KJ, et al., Global Burden of Disease (GBD) Disability Adjusted Life Years (DALYs) and Health Life Expectency (HALE) Collaborators. Global, regional, and national disability-adjusted life years (DALYs) for 306 diseases and injuries and healthy life expectancy (HALE) for 188 countries, 1990-2013: quantifying the epidemiological transition. Lancet 2015;386:2145-91.

2. Global Burden of Disease Study Collaborators. Global, regional, and national incidence, prevalence, and years lived with disability for 301 acute and chronic diseases and injuries in 188 countries, 19902013: a systematic analysis for the Global Burden of Disease Study 2013. Lancet 2015;386:743-800.

3. Fransen M, McConnell S, Harmer AR, et al. Exercise for osteoarthritis of the knee. Cochrane Database Syst Rev 2015;1: CD004376.

4. Sampath KK, Mani R, Miyamori T, et al. The effects of manual therapy or exercise therapy or both in people with hip osteoarthritis: a systematic review and meta-analysis. Clin Rehabil 2015. [Epub ahead of print]

5. Christensen R, Bartels EM, Astrup A, et al. Effect of weight reduction in obese patients diagnosed with knee osteoarthritis: a systematic review and meta-analysis. Ann Rheum Dis 2007;66:433-9.

6. Quintrec JL, Verlhac B, Cadet C, et al. Physical exercise and weight loss for hip and knee osteoarthritis in very old patients: a systematic review of the literature. Open Rheumatol J 2014;8:89-95.

7. Hochberg MC, Altman RD, April KT, et al. American College of Rheumatology 2012 recommendations for the use of nonpharmacologic and pharmacologic therapies in osteoarthritis of the hand, hip, and knee. Arthritis Care Res (Hoboken) 2012;64:465-74.

8. McAlindon TE, Bannuru RR, Sullivan MC, et al. OARSI guidelines for the non-surgical management of knee osteoarthritis. Osteoarthr Cartil 2014;22:363-88.

9. National Institute for Health and Care Excellence. Osteoarthritis: care and management in adults. London: Clinical Guideline CG177, 2014.

10. Nelson AE, Allen KD, Golightly YM, et al. A systematic review of recommendations and guidelines for the management of osteoarthritis: the chronic osteoarthritis management initiative of the U.S. bone and joint initiative. Semin Arthritis Rheum 2014;43:701-12.

11. Zhang W, Doherty M, Leeb BF, et al. EULAR evidence based recommendations for the management of hand osteoarthritis: report of a Task Force of the EULAR Standing Committee for International Clinical Studies Including Therapeutics (ESCISIT). Ann Rheum Dis 2007:66:377-88.

12. Fernandes L, Hagen KB, Bijlsma JW, et al. EULAR recommendations for the non-pharmacological core management of hip and knee osteoarthritis. Ann Rheum Dis 2013;72:1125-35.

13. American Academy of Orthopaedic Surgeons. Treatment of osteoarthritis of the knee: evidence-based guideline. 2nd edn. Rosemont, IL, USA, 2013. http://www.aaos.org/research/guidelines/ TreatmentofOsteoarthritisoftheKneeGuideline.pdf

14. Misso ML, Pitt VJ, Jones KM, et al. Quality and consistency of clinical practice guidelines for diagnosis and management of osteoarthritis of the hip and knee: a descriptive overview of published guidelines. Med J Aust 2008;189:394-9.

15. Basedow M, Williams $H$, Shanahan EM, et al. Australian GP management of osteoarthritis following the release of the RACGP guideline for the non-surgical management of hip and knee osteoarthritis. BMC Res Notes 2015;8:536.

16. AGREE Enterprise. AGREE (Appraisal of Guidelines for Research and Evaluation) Instrument. http://www.agreetrust.org/

17. Peat G, McCarney $R$, Croft $P$. Knee pain and osteoarthritis in older adults: a review of community burden and current use of primary health care. Ann Rheum Dis 2001;60:91-7.

18. Arthritis Australia. Time to Move: Osteoarthritis: Arthritis Australia. 2014. http://www.arthritisaustralia.com.au/index.php/reports/ time-to-move-arthritis-reports.html

19. Brand CA, Harrison C, Tropea J, et al. Management of osteoarthritis in general practice in Australia. Arthritis Care Res (Hoboken) 2014;66:551-8.

20. Runciman WB, Hunt TD, Hannaford NA, et al. CareTrack: assessing the appropriateness of health care delivery in Australia. Med J Aust 2012;197:100-5.

21. Cottrell E, Roddy E, Foster NE. The attitudes, beliefs and behaviours of GPs regarding exercise for chronic knee pain: a systematic review. BMC Fam Pract 2010;11:4.

22. Brand CA, Ackerman IN, Bohensky MA, et al. Chronic disease management: a review of current performance across quality of care domains and opportunities for improving osteoarthritis care. Rheum Dis Clin North Am 2013;39:123-43.

23. DeHaan MN, Guzman J, Bayley MT, et al. Knee osteoarthritis clinical practice guidelines-how are we doing? J Rheumatol 2007;34:2099-105.

24. Glazier RH, Dalby DM, Badley EM, et al. Management of common musculoskeletal problems: a survey of Ontario primary care physicians. CMAJ 1998;158:1037-40.

25. Kingsbury SR, Conaghan PG. Current osteoarthritis treatment prescribing influences and barriers to implementation in primary care. Prim Health Care Res Dev 2012;13:373-81.

26. Jawad AS. Analgesics and osteoarthritis: are treatment guidelines reflected in clinical practice? Am J Ther 2005;12:98-103.

27. Grol R. Successes and failures in the implementation of evidence-based guidelines for clinical practice. Med Care 2001;39 (Suppl 2):I146-54.

28. Hunter DJ. Quality of osteoarthritis care for community-dwelling older adults. Clin Geriatr Med 2010;26:401-17. 
29. Green LW. Making research relevant: if it is an evidence-based practice, where's the practice-based evidence? Fam Pract 2008;25 (Suppl 1):i20-4.

30. Balas EA, Boren SA. Managing clinical knowledge for health care improvement. In: Bemmel J, McCray AT, eds. Yearbook of Medical Informatics 2000: Patient-Centered Systems. Stuttgart, Germany: Schattauer Verlagsgesellschaft mbH; 2000:65-70.

31. Cabana MD, Rand CS, Powe NR, et al. Why don't physicians follow clinical practice guidelines? A framework for improvement. JAMA 1999;282:1458-65.

32. Miles MB, Huberman AM, Saldaña J. Qualitative data analysis: a methods sourcebook. 3rd edn. Los Angeles: Sage, 2014.

33. Strauss A, Corbin J. Basics in qualitative research: techniques and procedures for developing grounded theory. 2nd edn. Thousand Oaks, CA: Sage Publications, 1998.

34. Tong A, Flemming K, Mclnnes E, et al. Enhancing transparency in reporting the synthesis of qualitative research: ENTREQ. BMC Med Res Methodol 2012;12:181.

35. Noyes J, Hannes K, Booth A, et al. Chapter 20: qualitative research and Cochrane reviews. In: Higgins J, Green S, eds. Cochrane handbook for systematic reviews of interventions version 530 (updated October 2015). The Cochrane Collaboration, 2015:1-26.

36. Hannes K, Macaitis K. A move to more systematic and transparent approaches in qualitative evidence synthesis: update on a review of published papers. Qual Res 2012;12:402-42.

37. Barnett-Page E, Thomas J. Methods for the synthesis of qualitative research: a critical review. BMC Med Res Methodol 2009;9:59.

38. Moher D, Shamseer L, Clarke M, et al. Preferred reporting items for systematic review and meta-analysis protocols (PRISMA-P) 2015 statement. Syst Rev 2015;4:1.

39. Slade SC, Kent P, Bucknall T, et al. Barriers to primary care clinician adherence to clinical guidelines for the management of low back pain: protocol of a systematic review and meta-synthesis of qualitative studies. BMJ Open 2015;5:e007265.

40. Noyes J, Hannes K, Booth A, et al. on behalf of the Cochrane Qualitative and Implementation Methods Group. Chapter 20: Qualitative research and Cochrane reviews. In: Higgins JPT, Green $\mathrm{S}$, eds. Cochrane Handbook for Systematic Reviews of Interventions Version 5.3.0 (updated October 2015). The Cochrane Collaboration, 2015. http://qim.cochrane.org/supplemental-handbook-guidance
41. Higgins J, Green S, eds. Cochrane handbook for systematic reviews of interventions. Version 5.3.0 (updated October 2015) ed. The Cochrane Collaboration, 2015.

42. Critical Appraisal Skills Programme (CASP). CASP Qualitative Research Checklist: 10 questions to help you make sense of qualitative research. Oxford: Public Health Resource Unit; UK: Milton Keynes Primary Care Trust, 2002. http://media.wix.com/ugd/ dded87_29c5b002d99342f788c6ac670e49f274.pdf

43. Hannes K. Chapter 4: Critical appraisal of qualitative research. In: Noyes J, Booth A, Hannes K, Harden A, Lewin S, Lockwood C, eds. Supplementary Guidance for Inclusion of Qualitative Research in Cochrane Systematic Reviews of Interventions. Version 1 (updated August 2011). Cochrane Collaboration Qualitative Methods Group, 2011. http://cqrmg.cochrane.org/supplemental-handbook-guidance

44. Slade SC, Kent P, Patel S, et al. Barriers to primary care clinician adherence to clinical guidelines for the management of low back pain: a systematic review and meta-synthesis of qualitative studies. Clin J Pain 2015. In press.

45. Lewin $\mathrm{S}$, Glenton $\mathrm{C}$, Munthe-Kaas $\mathrm{H}$, et al. Using qualitative evidence in decision making for health and social interventions: an approach to assess confidence in findings from qualitative evidence syntheses (GRADE-CERQual). PLoS Med 2015;12: e1001895

46. French SD, Green SE, O'Connor DA, et al. Developing theory-informed behaviour change interventions to implement evidence into practice: a systematic approach using the Theoretical Domains Framework. Implement Sci 2012;7:38.

47. Michie S. Designing and implementing behaviour change interventions to improve population health. J Health Serv Res Policy 2008;13(Suppl 3):64-9.

48. Craig $\mathrm{P}$, Dieppe $\mathrm{P}$, Macintyre $\mathrm{S}$, et al. Developing and evaluating complex interventions: the new Medical Research Council guidance. BMJ 2008;337:a1655

49. Grol R, Wensing $M$. What drives change? Barriers to and incentives for achieving evidence-based practice. Med J Aust 2004;180: S57-60.

50. Baker R, Camosso-Stefinovic J, Gillies C, et al. Tailored interventions to overcome identified barriers to change: effects on professional practice and health care outcomes. Cochrane Database Syst Rev 2010;(3):CD005470. 